\title{
The mutually complementary role of magnetic resonance enterography and conventional enteroclysis in patients with complicated and/or advanced stage of Crohn's disease
}

\author{
Sanli DET ${ }^{1}$, Sanli AN ${ }^{3}$, Kandemirli $\mathrm{SG}^{3}$, Esmerer E${ }^{4}$, Kayadibi $\mathrm{Y}^{5}$, Demiryas $\mathrm{S}^{2}$, Korman $\mathrm{MU}^{6}$
}

Department of Radiology, Acibadem Hospitals Group, Istanbul, Turkey. tekcandenizesin@gmail.com

\begin{abstract}
AIM: To assess the diagnostic significance of magnetic resonance enterography (MRE) and conventional enteroclysis (CE) in patients with complicated and/or advanced stage of Crohn's disease.

METHODS: Patients with abnormal CE findings suggestive of mural and/or extramural involvement with the diagnosis or pre-diagnosis of CD are evaluated. After real-time bowel distension by enteroscopic examination, the patients with advanced or complicated stage were taken to the MRE examination in the same session. Mucosal-mural-extramural and activation findings, presence of stenosis/stricture, skip lesions and the mean duration of exams were evaluated with both CE and MRE. The superiority of one method over the other relative to these findings was evaluated.

RESULTS: A total of 110 patients evaluated by CE had the findings of CD. Of these, 24 patients with abnormal CE findings suggestive of advanced mural and extramural involvements were subsequently evaluated with MRE. CE was superior to MRE in the depiction of early superficial mucosal changes (aphthous-linear ulcer), cobblestone pattern $(p=0.002, p<0.01)$, obstruction $(p=0.004, p<0.01)$, and differentiation between the string sign and stricture. MRE was superior to conventional enteroclysis in mural and perienteric findings of bowel thickening, fibro-fatty proliferation, abscess $(p=0.016, p<0.05)$ and colonic skip lesions. No significant difference was found between the evaluated methods in terms of fistula detection $(p=1.000 ; p>0.05)$.

CONCLUSION: CE and MRE are mutually complementary imaging modalities in CD staging, evaluation of activation findings, and detection of complications (Tab. 3, Fig. 8, Ref. 23). Text in PDF www.elis.sk KEY WORDS: Crohn's disease, enteroclysis, magnetic resonance enterography, magnetic resonance imaging, complicated, mucosal, mural, extramural.
\end{abstract}

\section{Introduction}

Crohn's disease (CD) is a chronic inflammatory disease of the gastrointestinal tract, characterized by luminal, transmural, and mesenteric involvement, mainly at the terminal ileum (1). Although conventional enteroclysis (CE) used to be the cornerstone for evaluating the small intestine in $\mathrm{CD}$ in the previous decades, the new techniques like capsule endoscopy and double-balloon enteroscopy have allowed new ways of assessing the small bowel

${ }^{1}$ Department of Radiology, Acibadem Hospitals Group, Istanbul, Turkey, ${ }^{2}$ Department of General Surgery, Cerrahpaşa Faculty of Medicine, Istanbul University Cerrahpaşa, Istanbul, Turkey, ${ }^{3}$ Department of Radiology, Faculty of Medicine, The University of Iowa, Iowa City, US, ${ }^{4}$ Department of Radiology, Istanbul Esenler Gynecology and Pediatrics Hospital, Istanbul, Turkey, ${ }^{5}$ Department of Radiology, Istanbul Gaziosmanpasa Taksim Training and Research Hospital, Istanbul, Turkey, and ${ }^{6}$ Department of Radiology, Cerrahpaşa Faculty of Medicine, Istanbul University-Cerrahpaşa, Istanbul, Turkey

Address for correspondence: D.E.T. Sanli, Dr, 19 Mayis Mah. Inonu Cad. Okur Sok. No.24/A, Kozyatagi, Istanbul, Turkey.

Phone: +90.544 .8104446$
(2-4). Nevertheless, radiological methods are still important for both initial diagnosis and assessment of complications.

$\mathrm{CE}$ is a technique based on distending the small bowel with barium-methylcellulose suspension, which enables to detect mucosal changes and luminal abnormalities $(5,6)$. CE also gives func-

Tab.1. The evaluation of CDAI (Crohn's disease activity index).

\begin{tabular}{lc}
\hline Clinical or laboratory parameters & Impact factor \\
\hline Liquid or soft stools every day for seven days & x2 \\
Need for Lomotil or opiates for diarrhea & x30 \\
Poor general health conditions every day for seven days & x7 \\
(subjectively scored from 0 (good) to 4 (very bad)) & \\
Presence of complications * & x20 \\
Presence of abdominal mass (0 normal, 2 suspicious, 5 sure) & x10 \\
Hematocrit $<0.47$ in men and $<0.42$ in women & x6 \\
Weight loss & x1 \\
* 1 point is added for each complication group as follows: & \\
- Rheumatological findings (joint pain, i.e arthralgia or arthritis) & \\
- Eye findings (iritis or uveitis) & \\
- Skin findings (erythema nodosum, pyoderma gangrenosum or aphthous ulcers) \\
- Perianal findings (anal fissure, anal fistula or abscess) \\
- Other fistulas \\
- Fever during the previous week
\end{tabular}



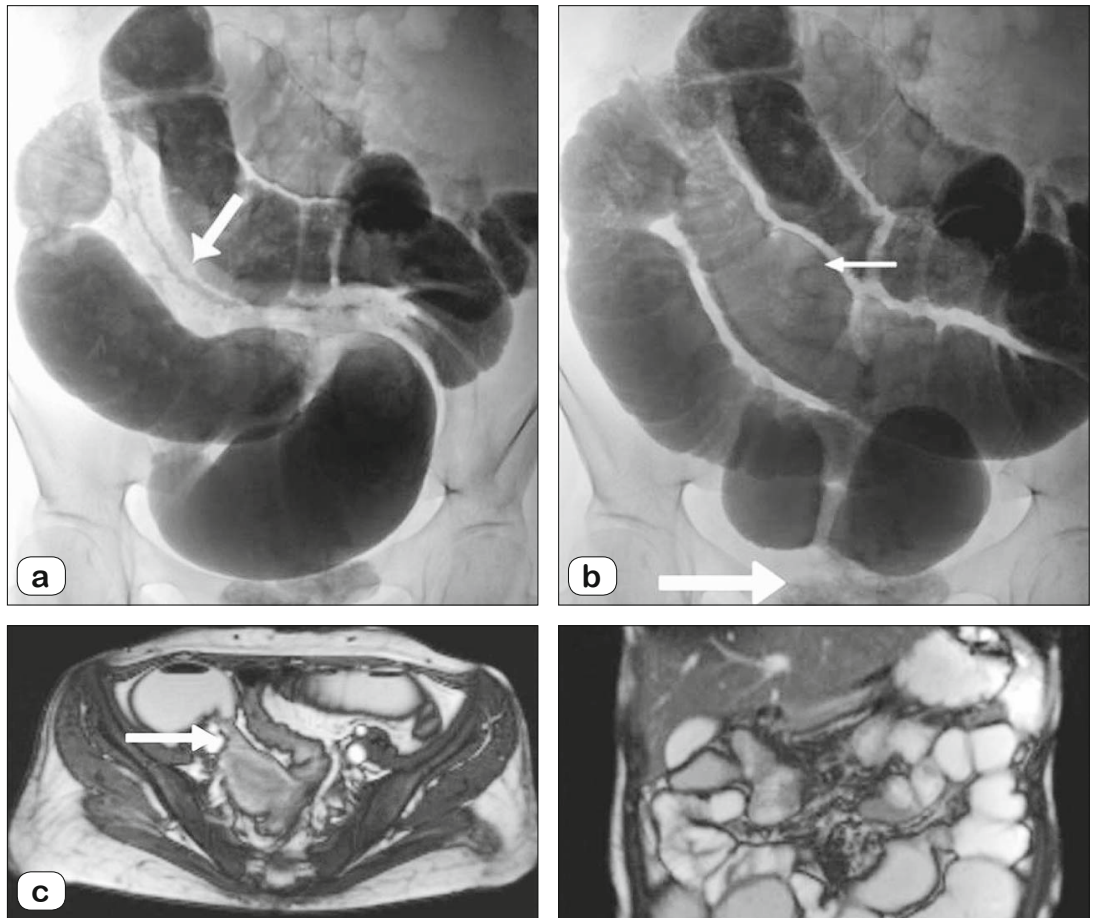

Fig. 1. There is a $25-\mathrm{cm}$ segment of collapsed lumen at mid-ileal segment (1.a, arrow) that re-expands with high hydrostatic pressure (1.b, thin arrow) on CE. Also note the opacification of rectum while right hemicolon is not opacified, suggestive of an enterocolic fistula (1.b, thick arrow). Subsequent MRE with axial and coronal T2-weighted images [true fast imaging with steady state free precession (TRUFI)] show only a diffuse wall thickening at these segments (1.c.d, arrow).
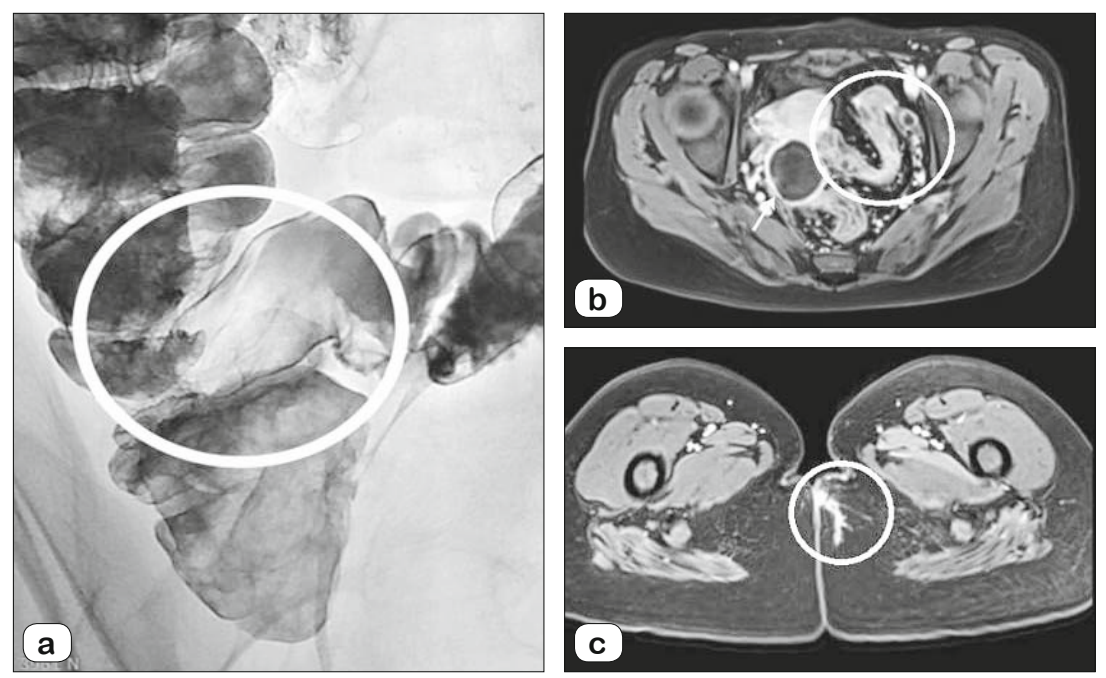

Fig. 2. Multiple small superficial ulcerations are noted on CE spot image (2.a, circle). Cobblestone pattern can be seen on axial fat suppressed contrast enhanced T1-weighted imaging (2.b, circle). Also note the abscess cavity with a regular rim-enhancing wall (2.b, arrow). More caudal images demonstrate the perianal fistula tract extending along the gluteal fat planes (2.c, arrow). tional information on the dynamic nature of the intestines, which allows the evaluation of bowel distensibility and fixation of bowel loops (6). However, CE gives only indirect information on the bowel wall and perienteric changes, and can be technically challenging due to the overlapping of bowel loops $(5,6)$. The age is an important feature to be considered in the management of younger patients due to accumulation of the radiation dose over time by repetitive imaging studies $(7,8)$. Also, the invasive nature of enteroclysis which requires intubation of the duodenum led to a wider adoption of enterography techniques (5-9). Advances in magnetic resonance imaging (MRI) techniques with faster image acquisitions allowed an assessment of small bowel by MRE (10). In addition to evaluating the luminal abnormalities, MRE allows to a limited degree to assess bowel wall and mesenteric changes (7-10). Although CE has been reported to achieve better luminal distention, in this respect there is paucity of data when compared with MRE. However, the general trend is the initial use of MRE while reserving enteroclysis for nondiagnostic enterography results (11-13). The aim of this study was to assess the success of detecting mucosal-mural-extramural findings, activation findings and complications of $\mathrm{CD}$ by both methods, MRE and CE. Additionally, we aimed to illustrate the imaging findings, and complications of CD to better understand the pathophysiology of the disease.

\section{Material and methods}

\section{Study population}

This was a prospectively designed study in patients with known or suspected CD based on clinical and laboratory findings. Between January 2013 and February 2015, 110 patients who had clinical and laboratory or imaging findings of CD were evaluated by CE. Of these, 24 patients who had abnormal CE findings suggestive of an advanced mural and extramural involvements such as fistula, abscess, and strictures were subsequently evaluated with MRE in the same session. The activity of CD was defined by a combination of clinical and laboratory scores. The Crohn's disease activity index $(\mathrm{CDAI})>150$ was considered as a state of clinically active disease (Tab. 1). 

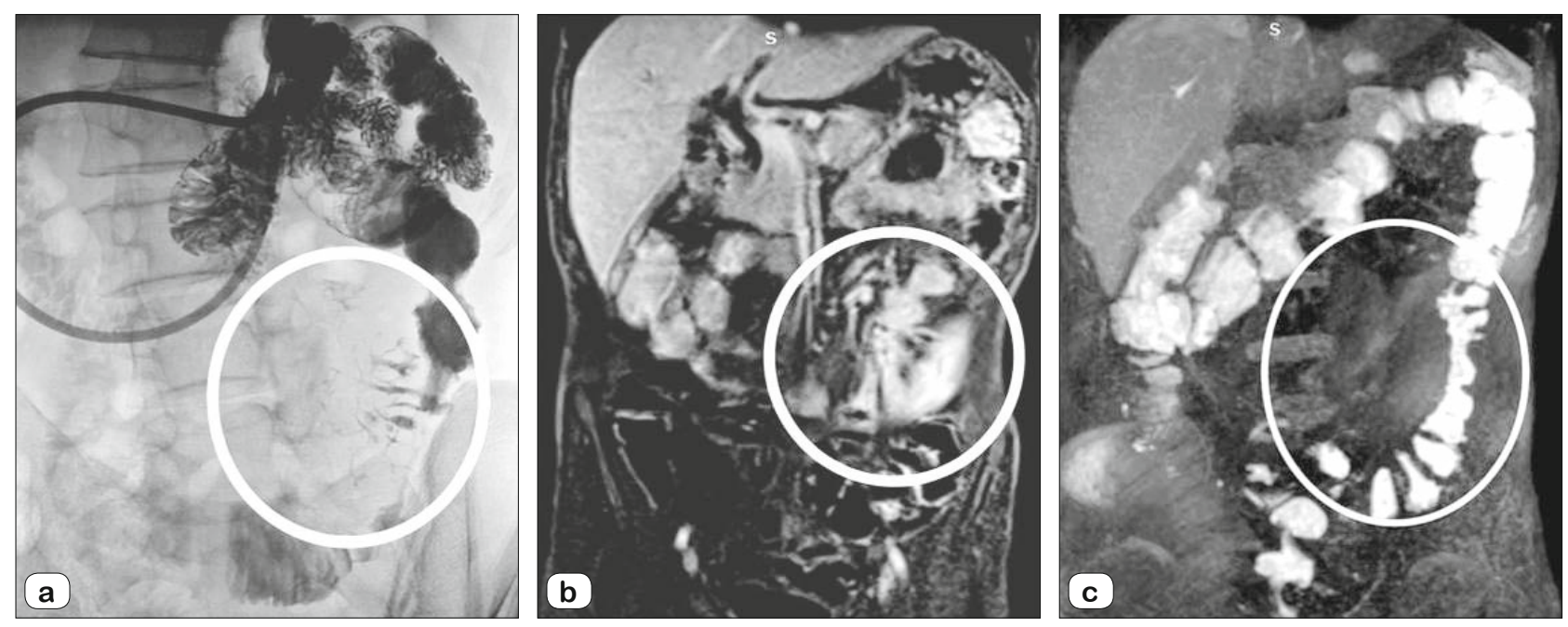

Fig. 3. CE demonstrates multiple high-flow fistulas at the level of proximal jejunum that extending medially with opacification of neighboring bowel loops (3.a, circle). Multiple fistulas with contrast enhancement along with bowel wall thickening and enhancement can be seen on MRE (3.b, circle). There is mesenteric rigidity with antimesenteric scalloping at descending colon with a $5 \mathrm{~cm}$ segment of narrowing due to disease involvement on coronal T2-weighted image (3.c, circle).
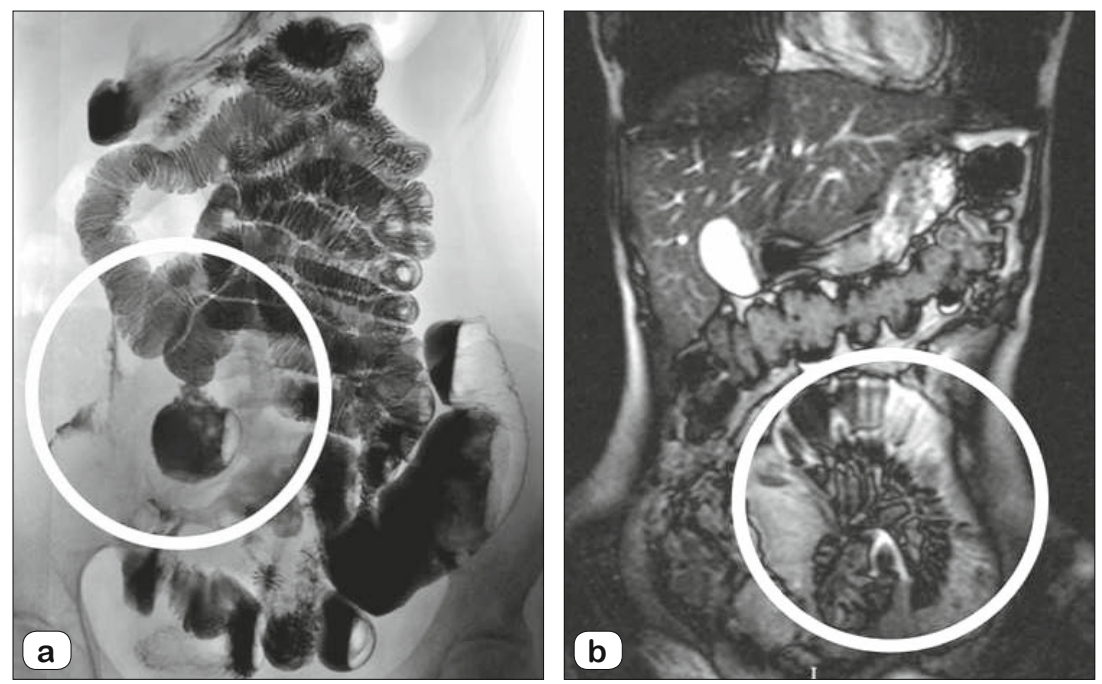

Fig. 4. The stenotic segment at terminal ileum with involvement of ileocecal valve and deformation at cecum and ascending colon can be seen on CE imaging (4.a, circle). Multiple entero-enteric fistulas, separation of this bowel segment from other bowel loops and opacification of an abscess pouch are also noted (4.a). On coronal fat suppressed contrast enhanced T1-weighted

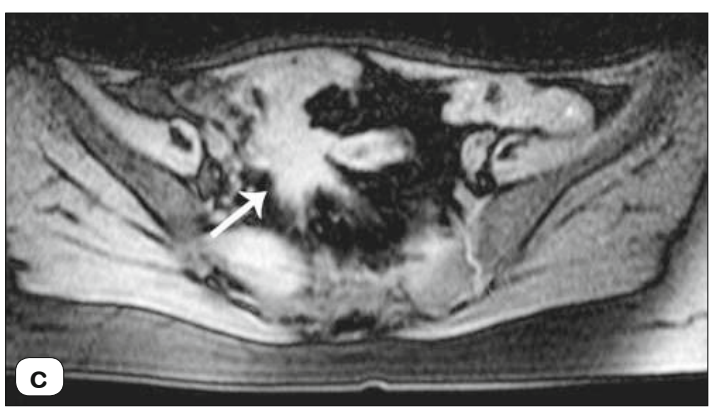

imaging, a comb sign due to increased mesenteric vascularity (4.b, circle) can be seen. Axial fat suppressed contrast enhanced T1-weighted image shows an abscess formation (4.c, arrow) with spiculated margins that extend into fat planes and neighboring bowel loops. This abscess is clearly demonstrated both on CE by opacification and on MRE as an enhancing spiculated mass.
The approval of ethics for this study was received from the local ethics committee (Decision No: 02-69909; Date: March 06, 2015).

\section{Conventional enteroclysis}

All CE procedures were performed based on the technique described by Herlinger (14). After transnasal intubation with a $12 \mathrm{~F}$ naso-enteric catheter (EZEM, Westbury, USA), the catheter tip was advanced just distal to the Treitz ligament. Initially, $50 \%$ barium suspension of 200 to $250 \mathrm{ml}$ was infused at a rate of 75 to $175 \mathrm{ml} / \mathrm{min}$. This was followed by infusion of a $0.5 \%$ methylcellulose suspension of 1,500 to 2,000 $\mathrm{ml}$ at a rate of 200 to $250 \mathrm{~mL} / \mathrm{min}$ to achieve double contrast. The infusion rate was modified when necessary based on the degree of bowel distension, peristaltic activity, and patients' tolerance. A C-arm digital angiography scope system (Artis Zee, Siemens Medical System, Germany) was used during the procedures. This method helped to take images from different angles and minimize limitations due to bowel superposition by rotating the $\mathrm{C}$ arm around the patient.

\section{MRE protocol}

After completing CE, the patients were given intravenous hyoscinebromide (Buscopan, Boehringer, Ingelheim, Germany) 
to decrease peristalsis and to prolong small bowel distention. Patients were immediately transferred to the MRI unit (1.5 Tesla Avanto, Siemens, Erlangen, Germany). No additional oral contrast material was necessary for MRE since bowels were optimally distended during CE. Also, methylcellulose suspension has biphasic contrast agent properties and acts as a positive contrast on true FISP images (fast imaging with steadystate free precession) and negative contrast on T1-WI. Gadoteric acid at a dose of 0.1 $\mathrm{mmol} / \mathrm{kg}$ was used as the intravenous contrast agent. Subjects were imaged in the supine position. An abdominal phased-array coil was used in all patients. The following sequences were obtained: standard 3 planes T1-weighted fast low-angle shot MRI (FLASH, TR: $1.42 \mathrm{~ms}$, TE: $2.72 \mathrm{~ms}$ ) and T1-weighted fat-saturated FLASH sequences (fast low-angle shot) without and with intravenous contrast; fast imaging with steady-state free precession (true FISP, TR: 4.5-5 ms, TE: 1.6-3.8 ms), and T2-weighted true fast imaging with steady-state free precession images (TRUFI, TR:595.36 ms, TE: $2.15 \mathrm{~ms}$ ) in coronal and axial planes. MRE images were obtained with a slice thickness of $4 \mathrm{~mm}$, and field of view of 230x80 mm.

\section{Image analysis}

$\mathrm{CE}$ and MRE images were evaluated by two radiologists who have experience in $\mathrm{CE}$ and cross-sectional examination. On CE, mucosal findings were evaluated according to the stage of the disease. The mucosal changes were categorized as superficial aphthous ulcers in the early stage, linear ulcers in the middle stage, or ulceronodular pattern, which gives the appearance of cobblestone in the advanced stage. During dynamic-scope imaging, motility disorders, degree of obstruction, and differentiation of pseudo-obstruction and strictures were assessed (Figs 1-6). Additionally, perienteric abnormalities such as fistula and mesenteric rigidity were assessed during dynamic scopic examination with $\mathrm{CE}$. Based on CE imaging findings, the patients were placed in a group of ulcerating, stenotic, or fistulating form. Mural and luminal abnormalities such as cobblestone pattern, degree and length of wall thickening were assessed on MRE. Perienteric findings such as fistula, mesenteric fatty proliferation, and abscess formation were evaluated on MRE. The wall thickness greater than $3 \mathrm{~mm}$ in an optimally distended bowel loop was defined as an abnormal wall thickening. On CE and MRE, the tulas (6.b, arrows).
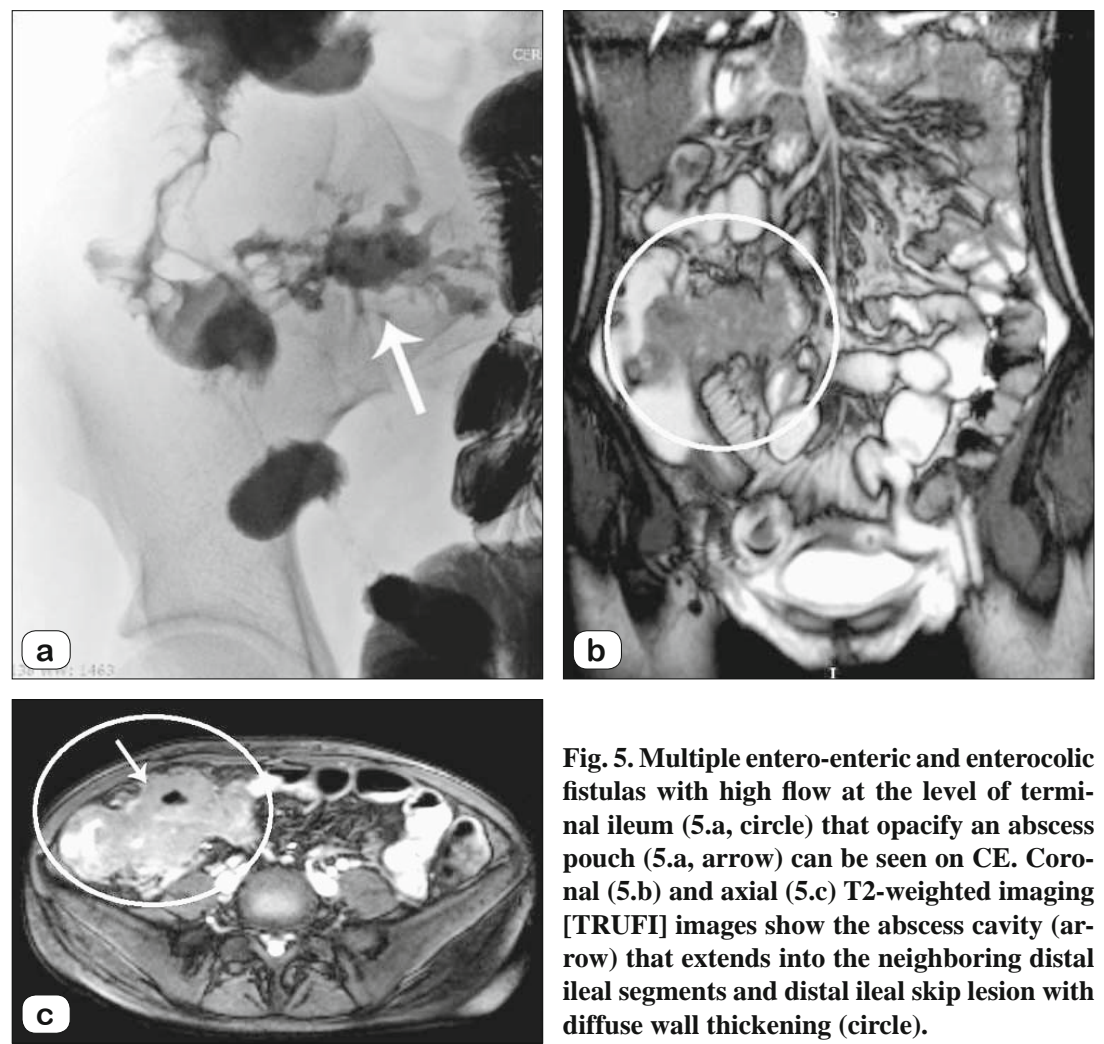

Fig. 5. Multiple entero-enteric and enterocolic fistulas with high flow at the level of terminal ileum (5.a, circle) that opacify an abscess pouch (5.a, arrow) can be seen on CE. Coronal (5.b) and axial (5.c) T2-weighted imaging [TRUFI] images show the abscess cavity (arrow) that extends into the neighboring distal ileal segments and distal ileal skip lesion with diffuse wall thickening (circle).

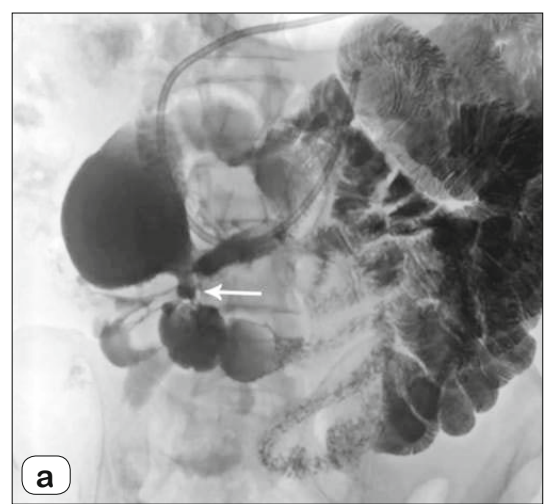

Fig. 6. CE shows multiple entero-enteric and enterocolic fistulas at the level of proximal ileal segment (6.a, arrow). Subsequent coronal T2-weighted image [TRUFI] also clearly demonstrates the entero-enteric and enterocolic fis-

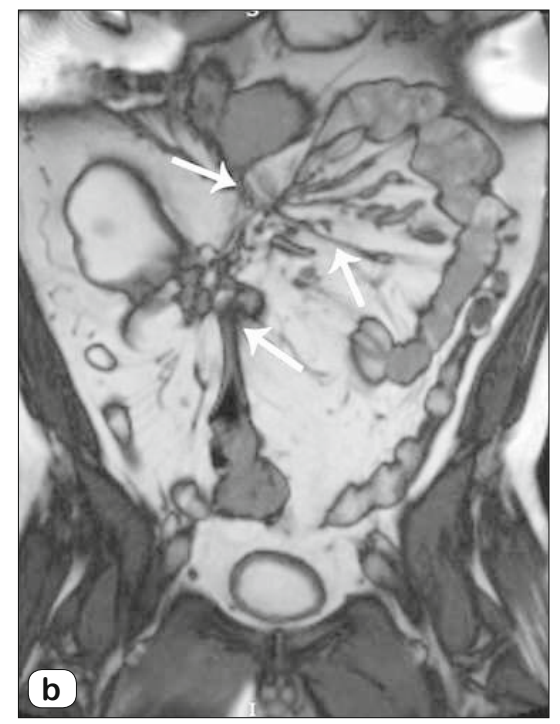

length of the involved bowel segment(s) was categorized as short $(\leq 5 \mathrm{~cm})$, medium $(5-15 \mathrm{~cm})$ or long $(\geq 15 \mathrm{~cm})$. Additionally, the presence of skip lesions was assessed with each of the methods. Skip lesions were categorized as either enteric or colonic.

\section{Statistical analysis}

NCSS (Number Cruncher Statistical System) program was used for statistical analysis. Descriptive statistical methods (fre- 


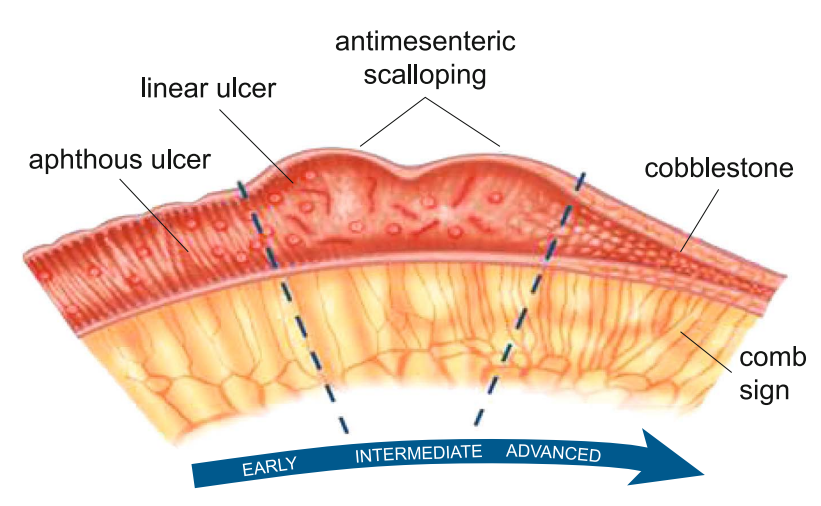

Fig. 7. Mucosal-mural-mesenteric changes in early-intermediate-advanced stage of Crohn's disease.

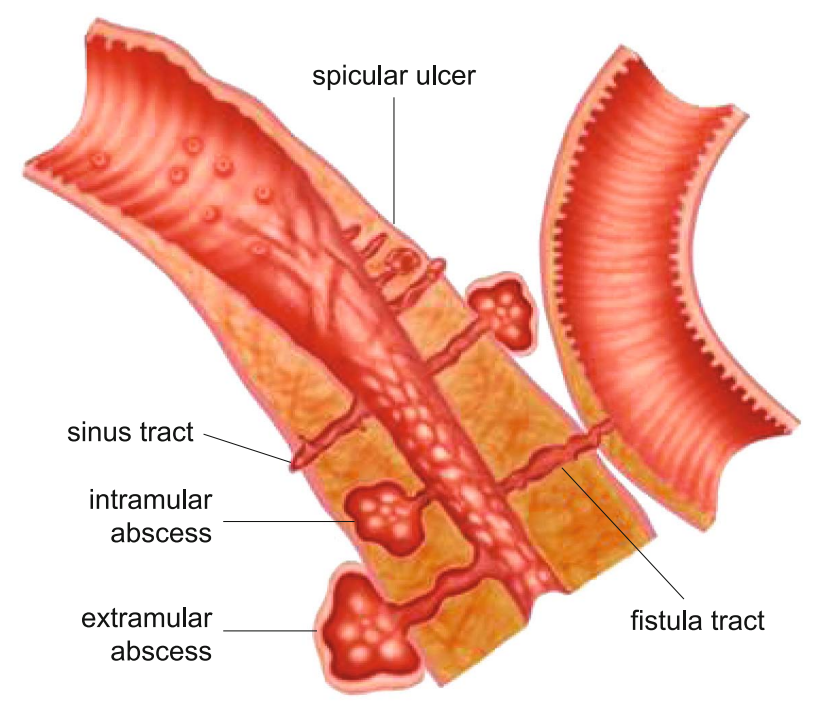

Fig. 8. Transmural Spread Patterns of Crohn's disease.

quency, percentage) were used while evaluating the study data. McNemar test and kappa goodness-of-fit-value were used to evaluate the agreement between $\mathrm{CE}$ and MR results for the diagnosis. Statistical significance was accepted at $\mathrm{p}<0.05$.

\section{Results}

This study included 24 advanced and/or complicated cases with a known or suspected diagnosis of $\mathrm{CD}$ who had signs of the mural and extramural extensions on CE. Of these 24 patients, 14 were female and 10 were male. The mean age of patients was 32.2 years $(18-60 \mathrm{y})$.

The mean duration of MRE was 10 minutes, while in enteroclysis, it was 25 minutes. Except for a single case of prior ileotransversostomy surgery, all cases had an involvement of terminal ileum. Of the total of 24 cases, MRE detected signs of activation such as mural enhancement, increased mesenteric vascularity, presence of mesenteric lymphadenopathy, and perienteric fluid in $18(75 \%)$ cases, and these findings were only detectable upon
MRE. CDAI was above 150 in 15 of these 18 patients with MRE findings of active inflammation. Of these 18 cases, CE detected signs of activation like aphthous-linear ulcers and string sign in 12 cases with a correlation level of $66 \%$. On CE, skip lesions were detected in 14 patients ( 11 enteric, 3 colonic; $58 \%$ ); whereas MRE showed only 8 of the enteric but also 4 colonic skip lesions. Three colonic skip lesions that could not be detected by CE were detected by MRE. There was no statistically significant difference between $\mathrm{CE}$ and MRE in detecting enteric and colonic skip lesions $(\mathrm{p}=0.250 ; \mathrm{p}>0.05 ; \kappa=0.743, \mathrm{p}=0.125 ; \mathrm{p}>0.05 ; \kappa=$ 0.515 , respectively). Superficial aphthous and linear ulcers were only detected by CE. On CE, 6 patients had superficial aphthous ulcers, 7 patients had linear ulcers and 3 patients had scarring ulcers. Additionally, 16 patients $(67 \%)$ had the cobblestone pattern of chronic $\mathrm{CD}$ on $\mathrm{CE}$. MRE was able to detect only 6 of 16 patients with the cobblestone pattern. In terms of detecting the cobblestone pattern, CE was statistically significantly superior to MRE ( $\mathrm{p}=$ $0.002, \mathrm{p}<0.01 ; \kappa=0.286)$. On CE, 6 patients $(25 \%)$ had a string sign, which was only detected by CE, whereas 14 patients $(58 \%)$ had strictures. Stricture was detected in 8 patients with MRE. CE was significantly superior to MRE in terms of detecting obstruction $(p=0.004 ; p<0.01 ; \kappa=0.341)$. On CE, 10 patients had fistula which included 4 cases of entero-enteric, 3 cases of enterocolonic, 2 cases of enterocutanoeus, and a single case of enterovesical fistula. Number, flow volume and location of fistula were assessed dynamically during scope imaging. Of the 10 fistula tracts detected on CE, 8 fistula tracts were also detected on MRE. There was no significant difference between the two evaluated methods in terms of fistula detection ( $p=1.000 ; p>0.05 ; \kappa=0.739)$. On MRE, 10 patients had abscess formation ( $42 \% ; 6$ intrabdominal, 2 subcutaneous, 2 intramural). CE was able to detect the intraabdominal abscess pouches in 3 out of 10 cases. In terms of detecting abscesses, MRE was significantly superior to CE ( $p=0.016$; $p<0.05, \kappa=0.333$ ). Based on imaging findings, the patients were placed in the group with ulcerating form ( 4 patients; $17 \%$ ), stenotic form (17 patients; $71 \%$ ), and fistulating form (3 patients; $12 \%$ ). The findings on CE and MRE are presented in Tables 2 and 3.

\section{Discussion}

In the past decade, several new endoscopy methods like push enteroscopy and capsule endoscopy have been developed for small bowel disease evaluation (2). Within the imaging realm, advances in fast MRE sequences have improved the image quality in MRE $(9,10,13,15)$. Several studies have shown that MRE provided competitive and/or complementary modalities compared to CE $(16,17)$. CE, which once used to be the principal imaging method for the evaluation of $\mathrm{CD}$, has fallen out of favor due to the advances in MRE. Many radiologists nowadays are unfamiliar with $\mathrm{CE}$ images, which we believe is crucial for proper evaluation and understanding of MRE findings.

$\mathrm{CE}$ is still superior to MRE in the evaluation of early mucosal changes $(6,7,16)$. Due to its dynamic evaluation, it can differentiate string sign from strictures that cannot be determined on static cross-sectional imaging $(6,16)$. Nevertheless, newer techniques that 
allow dynamic MR fluoroscopy can assess the severity of stenosis (18). CE has certain drawbacks such as overlapping of bowel segments that may hinder proper evaluation $(7,19)$. CE provides limited, indirect information on the transmural and perienteric changes $(7,20,21)$. This requires the use of complementary cross-sectional imaging like computed tomography or $\operatorname{MRE}(7,19$, $20)$. Early changes in CD are limited to the mucosal layer $(20,21)$. Lymphoid hyperplasia at the submucosa characterizes the initial changes in CD (20) (Fig. 7). On CE, this can be detected as subtle elevations and aphthous ulcers $(1,21)$. Due to its lower spatial resolution, magnetic resonance enteroclysis/enterography is inferior to $\mathrm{CE}$ in the detection of these early changes $(20,22)$. Aphthous ulcers in the early stages may progress to deeper linear ulcers or fissures (22). This can be detected as thin, high signal lines in a thickened bowel wall segment on MRE $(6,16,22)$. The cobblestone pattern defines a more chronic stage where there are patchy areas of involved and uninvolved segments seen as sharply demarcated high signal intensity areas on MRE. Deep ulcers may proceed to sinus and fistula formation $(18,22)$ (Fig. 8). Sinus tracts and fistulae are seen as high signal intensity tracts on fluid-sensitive sequences due to the fluid content, and as linear enhancing areas on fat-suppressed T1weighted images $(18,20,22)$. MRE allows to assess bowel wall thickening, as a thickness exceeding 4-5 $\mathrm{mm}$ in an optimally distended segment (18-20). Our study results suggest that MRE is clearly superior to CE in assessing perienteric changes and colonic findings of $\operatorname{CD}(7,18,20)$. The most common cause of bowel loop separation detected on CE is fibrofatty proliferation which can be easily detected with $\operatorname{MRE}(6,16,20)$. The second common cause is abscess formation, which can be detected with internal fluid content and contrast-enhancing rim with MRE (6, $7,20)$. Cross-sectional methods are needed to clarify the cause if intestinal separation is detected with CE. In accordance with the literature, our results indicate that $\mathrm{CE}$ is superior to MRE in the assessment of mucosal changes, obstruction, and differentiation between string sign and stricture $(6,19,20)$. However, it is well known that MRE is superior in the mural and perienteric findings such as fistula, abscess, fibrofatty prolifera-
Tab. 3. Statistical results of the findings according to both methods.

\begin{tabular}{|c|c|c|c|c|c|}
\hline & & \multicolumn{3}{|c|}{ "Aphthous Ulcer CE } & \multirow{3}{*}{$\mathrm{p}$} \\
\hline & & Negative & Positive & Total & \\
\hline & & $\mathrm{n}(\%)$ & $\mathrm{n}(\%)$ & $\mathrm{n}(\%)$ & \\
\hline \multirow{5}{*}{$\begin{array}{l}\text { Aphthous Ulcer } \\
\text { MRE }\end{array}$} & Negative & $18(75.0)$ & $\overline{60(25.0)}$ & $24(100.0)$ & \multirow[t]{3}{*}{-} \\
\hline & Positive & $0(0.0)$ & $0(0.0)$ & $0(0.0)$ & \\
\hline & Total & $18(75.0)$ & $6(25.0)$ & $24(100.0)$ & \\
\hline & & \multicolumn{3}{|c|}{ Linear ulcer CE } & \multirow[t]{2}{*}{$\mathrm{p}$} \\
\hline & & Negative & Positive & Total & \\
\hline \multirow[t]{5}{*}{ Linear ulcer MRE } & Negative & $17(70.0)$ & $7(29.2)$ & $24(100.0)$ & \multirow[t]{3}{*}{-} \\
\hline & Positive & $0(0.0)$ & $0(0.0)$ & $0(0.0)$ & \\
\hline & Total & $17(70.0)$ & $7(29.2)$ & $24(100.0)$ & \\
\hline & & \multicolumn{3}{|c|}{ Cobblestone pattern CE } & \multirow[t]{2}{*}{$\mathrm{p}$} \\
\hline & & Negative & Positive & Total & \\
\hline \multirow{5}{*}{$\begin{array}{l}\text { Cobblestone pattern } \\
\text { MRE }\end{array}$} & Negative & $8(33.3)$ & $10(41.7)$ & $18(75.0)$ & $0.002 * *$ \\
\hline & Positive & $0(0.0)$ & $6(25.0)$ & $6(25.0)$ & 0.286 \\
\hline & Total & $8(33.3)$ & $16(66.7)$ & $24(100.0)$ & \\
\hline & \multicolumn{5}{|c|}{ Wall thickening MRE } \\
\hline & & Negative & Positive & Total & \\
\hline \multirow{5}{*}{$\begin{array}{l}\text { Wall Thickening } \\
\text { CE }\end{array}$} & Negative & $0(0.0)$ & $9(37.5)$ & $9(37.5)$ & - \\
\hline & Positive & $0(0.0)$ & $15(62.5)$ & $15(62.5)$ & \\
\hline & Total & $0(0.0)$ & $24(100.0)$ & $24(100.0)$ & \\
\hline & & & struction C & & \\
\hline & & Negative & Positive & Total & \\
\hline Obstruction MRE & Negative & $7(29.2)$ & $9(37.5)$ & $16(66.7)$ & $0.004 * *$ \\
\hline & Positive & $0(0.0)$ & $8(33.3)$ & $8(33.3)$ & 0.341 \\
\hline & Total & $7(29.2)$ & $17(70.8)$ & $24(100.0)$ & \\
\hline & & & Fistula CE & & \\
\hline & & Negative & Positive & Total & \\
\hline Fistula MRE & Negative & $13(54.2)$ & $2(8.3)$ & $15(62.5)$ & 1.000 \\
\hline & Positive & $1(4.2)$ & $8(33.3)$ & $9(37.5)$ & 0.739 \\
\hline & Total & $14(58.3)$ & $10(41.7)$ & $24(100.0)$ & \\
\hline & & & bscess MR & & \\
\hline & & Negative & Positive & Total & \\
\hline Abscess CE & Negative & $14(58.3)$ & $7(29.2)$ & $21(87.5)$ & $0.016^{*}$ \\
\hline & Positive & $0(0.0)$ & $3(12.5)$ & $3(12.5)$ & 0.333 \\
\hline & Total & $14(58.3)$ & $10(41.7)$ & $24(100.0)$ & \\
\hline & & & nhancemen & & \\
\hline & & Negative & Positive & Total & \\
\hline Mural enhancement & Negative & $18(75.0)$ & $6(25.0)$ & $24(100.0)$ & - \\
\hline $\mathrm{CE}$ & Positive & $0(0.0)$ & $0(0.0)$ & $0(0.0)$ & \\
\hline & Total & $18(75.0)$ & $6(25.0)$ & $24(100.0)$ & \\
\hline & & & ic vascular & & \\
\hline & & Negative & Positive & Total & \\
\hline Mesenteric & Negative & $16(66.7)$ & $8(33.3)$ & $24(100.0)$ & - \\
\hline vascularity $\mathrm{CE}$ & Positive & $0(0.0)$ & $0(0.0)$ & $0(0.0)$ & \\
\hline & Total & $16(66.7)$ & $8(33.3)$ & $24(100.0)$ & \\
\hline & & Mes & ymphadeno & ARE & \\
\hline & & Negative & Positive & Total & \\
\hline Mesenteric & Negative & $18(75.0)$ & $6(25.0)$ & $24(100.0)$ & - \\
\hline lymphadenopathy & Positive & $0(0.0)$ & $0(0.0)$ & $0(0.0)$ & \\
\hline $\mathrm{CE}$ & Total & $18(75.0)$ & $6(25.0)$ & $24(100.0)$ & \\
\hline & & & teric fluid & & \\
\hline & & Negative & Positive & Total & \\
\hline Mesenteric fluid CE & Negative & $19(79.2)$ & $5(20.8)$ & $24(100.0)$ & - \\
\hline & Positive & $0(0.0)$ & $0(0.0)$ & $0(0.0)$ & \\
\hline & Total & $19(79.2)$ & $5(20.8)$ & $24(100.0)$ & \\
\hline & & & c skip lesio & & \\
\hline & & Negative & Positive & Total & \\
\hline Enteric skip lesion & Negative & $13(54.2)$ & $3(12.5)$ & $16(66.7)$ & 0.250 \\
\hline MRE & Positive & $0(0.0)$ & $8(33.3)$ & $8(33.3)$ & 0.743 \\
\hline & Total & $13(54.2)$ & $11(45.8)$ & $24(100.0)$ & \\
\hline & & & ic skip lesic & & \\
\hline & & Negative & Positive & Total & \\
\hline Colonic skip lesion & Negative & $17(70.8)$ & $4(16.4)$ & $21(87.5)$ & 0.125 \\
\hline MRE & Positive & $0(0.0)$ & $3(12.3)$ & $3(12.5)$ & 0.515 \\
\hline & Total & $17(70.8)$ & $7(29.2)$ & $24(100.0)$ & \\
\hline
\end{tabular}

McNemar Test 
tion, and colonic skip lesions $(1,6,7,19,20)$. Although there was no statistically significant difference between the methods in terms of fistula and skip lesion detection in our study, the ability of MRE to detect abscess was superior to $\mathrm{CE}$ in accordance with the literature. In our study, the methylcellulose solution used during $\mathrm{CE}$ acted also in the double contrast phase of the $\mathrm{CE}$ and provided a homogeneous bowel distension. Methylcellulose has also biphasic contrast agent properties and acts as positive contrast on true FISP images and negative contrast on T1-weighted imaging (23). For these reasons, the combination of CE and supplementary MRE offers the advantages of two different techniques in a less time-consuming method.

The limitations of our study included a selection bias where only patients with findings suggestive of mural-perienteric abnormalities were subsequently imaged with MRE. Thus, we excluded patients in early stages of CD from our study. Another limitation of our study may lie in the radiation exposure with CE. However, it is possible to get rid of this effect with minimum damage by adjusting the device calibration settings regularly and appropriately and also, by device signaling when a certain upper limit dose is exceeded. In our study, the duration of enteroclysis was longer than that of MRE. The reason for this can be explained by the fact that the cases were complicated with stenosis-stricture, which prolongs the passage time due to obstruction.

In conclusion, although $\mathrm{CE}$ has been largely replaced by MRE, each technique has its own superiorities and disadvantages. CE can depict early superficial mucosal changes, differentiate the string sign from stricture, and detect low-flow fistula due to its high spatial resolution and dynamic nature. MRE is superior in the detection of mural and perienteric findings of bowel thickening, fibrofatty proliferation, abscess, and skip lesions. MRE with its soft tissue contrast, multi-planar imaging, and lack of radiation exposure is an adequate imaging modality by itself in the assessment of CD complications. CE and MRE are imaging modalities that are mutually complementary in staging, evaluation of activation findings, and complications of CD.

\section{References}

1. Lopes S, Andrade P, Afonso J, Cunha R, Rodrigues-Pinto E, Ramos I, Macedo G, Magro F. Monitoring Crohn's disease activity: endoscopy, fecal markers and computed tomography enterography. Therp Adv Gastroenterol 2018; 11: 1756284818769075. DOI: 10.1177/1756284818769075.

2. Yang DH, Keum B, Jeen YT. Capsule Endoscopy for Crohn's Disease: Current Status of Diagnosis and Management. Gastroenterol Res Pract 2016; 2016: 8236367. DOI: 10.1155/2016/8236367.

3. Huang Z, Liu X, Yang F, Wang G, Ge N, Wang S, Guo J, Sun S. Diagnostic efficacy of double-balloon enteroscopy in patients with suspected isolated small bowel Crohn's disease. BMC Gastroenterol 2020; 20 (1): 42. DOI: $10.1186 / \mathrm{s} 12876-020-01188-0$.

4. Pennazio M, Spada C, Eliakim R et al. Small-bowel capsule endoscopy and device-assisted enteroscopy for diagnosis and treatment of small-bowel disorders: European Society of Gastrointestinal Endoscopy (ESGE) Clinical Guideline. Endoscopy 2015; 47 (4): 352-376. DOI: 10.1055/s-0034-1391855.

5. Maataoui A, Vogl TJ, Jacobi V, Khan MF. Enteroclysis: Current clinical value. World J Radiol 2013; 5 (7): 253-258. DOI: 10.4329/wjr.v5.i7.253. PMID: 23908695;
6. Akman C, Korman U, Ogut G et al. A combination of small bowel imaging methods: conventional enteroclysis with complementary magnetic resonance enteroclysis. Clin Radiol 2005; 60: 778-786.

7. Masselli G, Vecchioli A, Gualdi GF. Crohn disease of the small bowel: MR enteroclysis versus conventional enteroclysis. Abdom Imaging 2006; 31 (4): 400-409. DOI: 10.1007/s00261-005-0395-4.

8. Estay C, Simian D, Lubascher J, Figueroa C, O’BrienA, Quera R. Ionizing radiation exposure in patients with inflammatory bowel disease: are we overexposing our patients? J Dig Dis 2015; 16(2): 83-89. DOI: 10.1111/1751-2980.12213.

9. Fidler J. MR imaging of the small bowel. Radiol Clin North Am 2007; 45: 317-331.

10. Prassopoulos P, Papanikolaou N, Grammatikakis J, Rousomoustakaki M, Maris T, Gourtsoyiannis N. MR enteroclysis imaging of Crohn disease. Radiographics 2001; 21 Spec No: S161-172. DOI: 10.1148/radiographics.21. suppl_1.g01oc02s161.

11. Arrive L, El Mouhadi S. MR enterography versus MR enteroclysis. Radiology 2013; 266: 688.

12. Negaard A, Paulsen V, Sandvik L et al. A prospective randomized comparison between two MRI studies of the small bowel in Crohn's disease, the oral contrast method and MR enteroclysis. Eur Radiol 2007; 17: 2294-2301.

13. Masselli G, Gualdi G. MR imaging of the small bowel. Radiology 2012; 264: 333-348.

14. Herlinger $\mathbf{H}$. A modified technique for the double-contrast small bowel enema. Gastrointest Radiol 1978; 3: 201-207.

15. Fujii T, Naganuma M, Kitazume Y, Saito E, Nagahori M, Ohtsuka K, Watanabe M. Advancing magnetic resonance imaging in Crohn's disease. Digestion 2014; 89 (1): 24-30. DOI: 10.1159/000356210.

16. Korman U, Kurugoglu S, Ogut G. Conventional enteroclysis with complementary MR enteroclysis: a combination of small bowel imaging. Abdom Imaging 2005; 30: 564-575.

17. Umschaden HW, Szolar D, Gasser J, Umschaden M, Haselbach H. Small-bowel disease: comparison of MR enteroclysis images with conventional enteroclysis and surgical findings. Radiology 2000; 215: 717-725.

18. Scharitzer M, Ba-Ssalamah A. Moderne MRT des Dünndarms [Modern MRI of the small bowell]. Radiologe 2015 Dec; 55(12): 1067-1076. German. DOI: $10.1007 / \mathrm{s} 00117-015-0034-1$.

19. Sailer J, Peloschek P, Schober E, Schima W, Reinisch W, Vogelsang H, Wunderbaldinger P, Turetschek K. Diagnostic value of CT enteroclysis compared with conventional enteroclysis in patients with Crohn's disease. AJR Am J Roentgenol 2005; 185(6): 1575-1581. DOI: 10.2214/AJR.04.1534.

20. Gourtsoyiannis NC, Grammatikakis J, Papamastorakis G, Koutroumbakis J, Prassopoulos P, Rousomoustakaki M, Papanikolaou N. Imaging of small intestinal Crohn's disease: comparison between MR enteroclysis and conventional enteroclysis. Eur Radiol 2006; 16 (9): 1915-1925. DOI: $10.1007 / \mathrm{s} 00330-006-0248-8$.

21. Carbo AI, Reddy T, Gates T, Vesa T, Thomas J, Gonzalez E. The most characteristic lesions and radiologic signs of Crohn disease of the small bowel: air enteroclysis, MDCT, endoscopy, and pathology. Abdom Imaging 2014; 39 (1): 215-134. DOI: 10.1007/s00261-013-0036-2.

22. Scharitzer M, Koizar B, Vogelsang H, Bergmann M, Primas C, Weber M, Schima W, Mang T. Crohn's disease: prevalence, MR features, and clinical significance of enteric and colonic sinus tracts. Eur Radiol 2020; 30 (10): 5358-5366. DOI: 10.1007/s00330-020-06935-1.

23. Evrimler S, Algin O. MR enterography with oral contrast agent composed of methylcellulose, low-dose barium sulfate, sorbitol, and lactulose: assessment of diagnostic performance, reliability, image quality, and patient tolerance. Clin Imaging 2016; 40 (3): 523-530. DOI: 10.1016/j.clinimag.2016.01.002.

Received September 22, 2020. Accepted November 9, 2020. 